\title{
Incidence, Clinical Characteristics, and Histopathological Results of Atypia of Undermined Significance in a Tertiary Center in UAE
}

\author{
Maha Osman Shangab ${ }^{a}$ Azza Abdulaziz Khalifa ${ }^{b}$ Fatheya Al Awadi ${ }^{b}$ \\ Mouza Alsharhan ${ }^{c}$ Alaaeldin Bashier ${ }^{b}$ \\ anternal Medicine Department, Rashid Hospital, Dubai Health Authority, Dubai, UAE; bendocrinology Department, \\ Dubai Hospital, Dubai Health Authority, Dubai, UAE; 'Pathology Department, Dubai Hospital, Dubai Health Authority, \\ Dubai, UAE
}

\section{Keywords}

Thyroid nodule · Atypia of undetermined significance ·

Papillary thyroid cancer $\cdot$ Follicular thyroid cancer .

Bethesda $\cdot$ Fine needle aspiration

\begin{abstract}
Introduction: Bethesda classification of thyroid nodules cytology is an agreed upon method of classifying thyroid nodules according to risk of malignancy. Among them, Bethesda class III (Atypia of Undetermined Significance, AUS) has been a topic of great controversy. The reported estimated risk of malignancy associated with it varies in different studies. Our study investigates the associated incidence of malignancy in a single tertiary center in UAE. Methodology: Data were retrospectively collected over a 10-year period from January 2009 till December 2018 for cytology diagnosis of AUS. Patient charts were reviewed for method of management. Surgical and histopathology records were reviewed to compare findings on cytology versus histopathology. Results: A total of 180 cases were diagnosed Bethesda III (AUS) with a mean age of $45.09 \pm 14.7$ years. One hundred cases $(55.6 \%)$ of them underwent surgical resection, and histopathological diag-
\end{abstract}

karger@karger.com www.karger.com/dde

Karger!"

BOPEN ACCESS
(C) 2021 The Author(s)

Published by S. Karger AG, Basel

This article is licensed under the Creative Commons AttributionNonCommercial-NoDerivatives 4.0 International License (CC BYNC-ND) (http://www.karger.com/Services/OpenAccessLicense) Usage and distribution for commercial purposes as well as any distribution of modified material requires written permission. nosis was obtained. Among the operated cases, 46 were benign and 54 were malignant. Papillary thyroid cancer was the most common malignancy, seen in 39 (72.2\%) of cases, and follicular cancer was the second most common, seen in 13 (24.1\%) cases. Conclusion: The findings highlighted in our study suggest a higher incidence of malignancy in Bethesda III category than previously reported. It also puts in question the utility and benefit behind keeping a time gap and repeating FNA as previously recommended.

(c) 2021 The Author(s)

Published by S. Karger AG, Basel

\section{Introduction}

Thyroid nodules are common finding in general population, being found in up to $68 \%$ of general population by ultrasound or on autopsy [1]. The thyroid nodules and their associated risk of malignancy have been a topic of interest, with many studies assessing the utility of cytopathological analysis as initial triaging method $[2,3]$.

After the National Cancer Institute (NCI) thyroid fine needle aspiration (FNA) state of the science conference in 
2007, a new classification project was initiated [2]. The Bethesda System for Reporting Thyroid Cytopathology (TBSRTC) aims at unifying the terms and classifications to allow ease of communicating the findings in FNA cytopathology [2].

TBSRTC classifies thyroid nodules into 6 categories: non-diagnostic or unsatisfactory (Bethesda I), benign (Bethesda II), atypia/follicular lesion of undetermined significance (Bethesda III), follicular neoplasm/suspicion for a follicular neoplasm (Bethesda IV), suspicious for malignancy (Bethesda V), and malignant (Bethesda VI). Malignant lesions includes papillary thyroid carcinoma, poorly differentiated carcinoma, medullary thyroid carcinoma, anaplastic carcinoma, squamous-cell carcinoma, carcinoma with mixed features, metastatic carcinoma, non-Hodgkin lymphoma, and others [3]. Since their reporting, each Bethesda category is associated with a certain risk of malignancy, ranging between 0 and 3\% for Bethesda II and almost 100\% for Bethesda VI [3].

Among all the categories, Bethesda III remains a controversial cytopathological diagnosis [4]. It is defined based on the following cytopathogical features: lesions with mild nuclear atypia, atypical cysts lining cells, microfollicular lesions, extensive oncocytic (Hurthle cell) change, and specimens that are compromised or not otherwise specified. TBSRTC considered this category as a last resort for classification and suggested that it should not be more than $7 \%$ of total thyroid cytopathological results [3].

The associated risk of malignancy in this category was recently updated in 2017 with wide variations in the risk depending on the presence or absence of noninvasive follicular thyroid neoplasm with papillary-like nuclear features $(10-30 \%$ and $6-18 \%$ respectively) [3]. Other studies, however, show more heterogeneous associated risks of malignancy. In a meta-analysis done in 2012, a total 25,445 cases of thyroid nodules that underwent FNAs were analyzed. Bethesda category III reported rate was $9.6 \%$. Almost $40 \%$ of them underwent surgical resection, with a $15.9 \%$ incidence of malignancy [4].

Findings in the Middle Eastern population are inconsistent when it comes to the risk of malignancy in Bethesda III. A recent study done in 2018 in Saudi Arabia showed a calculated risk of malignancy reaching 50\% [5], whereas a similar study done in Bahrain revealed a drastically lower incidence of malignancy, of 9\% in Bethesda III [6]. Local data are lacking; for this reason, this retrospective cohort study was conducted to determine the risk of malignancy in Bethesda III, and hence improve clinical judgment and future management.

\section{Methods and Materials}

\section{Aim of the Study}

We aimed to assess the risk of malignancy in Bethesda III or atypia of undetermined significance (AUS) cytology patients in a single tertiary center in Dubai over a period of 10 years.

\section{Subjects and Methods}

After obtaining an institutional approval to collect the data for this study, we retrospectively examined a cohort of patients at Dubai Hospital, Dubai, United Arab Emirates, in the period between 1st of Jan 2009 and 31st December 2018. Data were collected from pathology department database using the terms (AUS), and Bethesda III. Data were collected and entered in an excel sheet; these included patient's demographics, ultrasound features of biopsied nodules, cytology report based on Bethesda classification, details of the histopathology report, and type and extent of surgery.

\section{Data Analysis}

Simple analysis using means $\pm S D$ as well as frequency analysis was used. Z-test was used to determine the significance difference between means and $\chi^{2}$ tests were used to assess the association between malignancy and the demographic variables in AUS cohort. Statistical significance was considered if the $p$-values $\leq 0.05$. Analyses were performed using SPSS software version 23 .

\section{Results}

Out of all patients who underwent FNA in our center over the past 10 years, 180 patients were diagnosed with Bethesda III (AUS or follicular lesion). Males were a minority $17.2 \%(n=31)$, while females were $82.8 \%(n=149)$. The mean age for the studied group was $45.09 \pm 14.7$. Minimum age was 15 years and maximum 80 years. Emirati patients were the majority and constituted 55\% ( $n=$ 99), followed by other Arab countries $23.3 \%(n=42)$; the number of patients from India and Indian subcontinent was equal, $6.7 \%(n=12)$ each. The remaining $8.3 \%(n=$ 15) were from either Far East, Europe, Africa, or Americas.

Out of 180 patients diagnosed as Bethesda III, 8 patients had biopsies taken from more than 1 nodule, and both nodules showed AUS or FLUS, 4.4\%. 74.4\% ( $n=138$ patients) were AUS, while $21.1 \%(n=38)$ were either follicular neoplasm or Hurthle cell neoplasm. Out of 180 patients, $55.6 \%(n=100)$ underwent thyroid surgery [see Fig. 1]. Details of the surgery were available for $78 \%(n=$ 78 ) of patients. Of those, $10.3 \%$ ( $n=8$ out of 78$)$ underwent thyroidectomy with lymph node dissection, $64.1 \%$ ( $n=50)$ underwent thyroidectomy, and the remaining $25.6 \%(n=20)$ underwent lobectomy.

The histopathology reports confirmed benign pathology in 46 out of 100 patients (46\%), while the rest were 


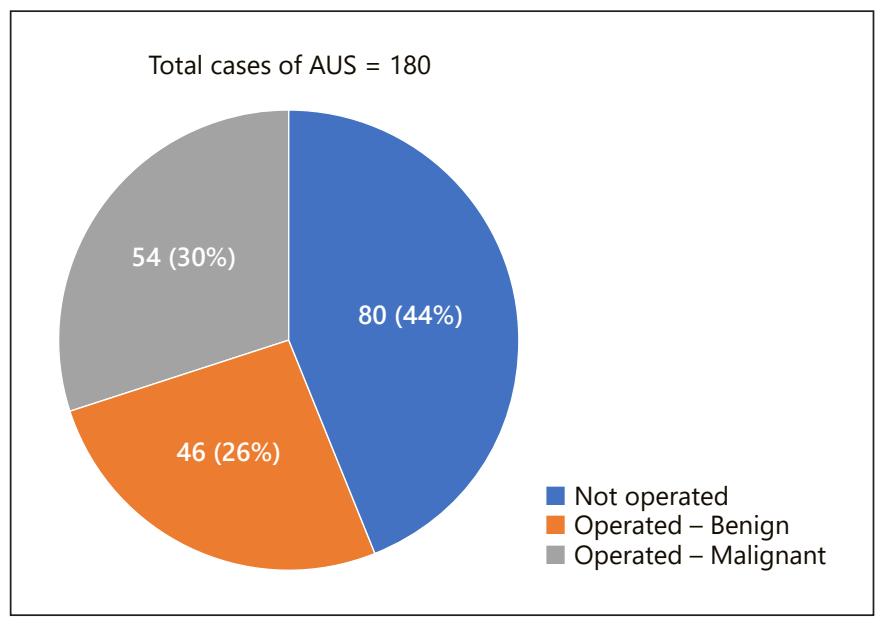

Fig. 1. Number of cases that had thyroid surgery and their outcomes.

diagnosed as thyroid cancer, 54 out of 100 patients (54\%). All types of thyroid cancer were seen. Papillary thyroid cancer was seen in 39 patients $72.2 \%$ of all cancers, while follicular cancer was seen in 13 patients (24.1\%). Two were diagnosed with Hurthle cell carcinoma (3.7\%). One patient was diagnosed with non-Hodgkin's lymphoma (1.6\%). There was one anaplastic (1.6\%) case and no cases of medullary thyroid cancer. Of note, the number of patients is 54, while the number of thyroid cancers is 56; this is explained by the fact that one patient has 2 synchronous tumors in 2 different nodules: one was papillary, while the second was follicular carcinoma. The second patient had mixed papillary/follicular thyroid cancer in the same nodule. The median size of the tumors was 1.25 $\mathrm{cm}$, and the range was between 0.1 and $8 \mathrm{~cm}$.

Data on focality was available for a small number of patients $(n=45) ; 32$ patients $(71 \%)$ were unifocal, while the rest were multifocal. Capsular invasion was seen in $48.9 \%(n=22)$, lympho-vascular invasion was seen in 10 patients $22.2 \%$, and lymph node metastasis was seen in only $13.3 \%(n=6)$.

\section{Discussion}

Thyroid cancer is the most common endocrine malignancy worldwide [7]. Diagnosing thyroid cancer in a patient presenting with thyroid nodule is done through an algorithm that starts with thyroid FNA cytology. Cytopathological findings since 2007 have been reported worldwide using the TBSRTC in order to facilitate communica- tion and unify terminology [3]. TBSRTC however has left the scientific community with a clinical controversy regarding the clinical significance and associated risk of malignancy of Bethesda III. Our current study highlights the epidemiological findings and associated risk of malignancy in UAE population concerning the group of patients with thyroid nodules with Bethesda III.

The incidence of thyroid nodules is generally higher in females compared to males [8]. Among the 180 cases involved in our study, females were the majority, $82.8 \%$ $(n=149)$. This finding is similar to recent studies done in Finland [9], Macedonia [10], and North America [11, 12]. The age group in our study was around $45.09 \pm 14.7$, which was comparable to a study in Thailand [13], but it was a decade younger than what was reported in other studies [10-12]. Emirati patients were the majority and constituted $55 \%(n=99)$. As a result, data in this study can, to a certain extent, be extrapolated to the whole local population.

The recommended management for Bethesda category III is to repeat FNA unless there are suspicious clinical or sonographic features, abnormal repeated aspiration, or abnormal molecular testing results, in which surgical resection would be the appropriate next step [3]. In our study, 100 (55.6\%) of our cases underwent surgical resection due to presence of clinical and radiological risk factors. This proportion is similar to the management done in other studies $[12,14]$.

According to TBSRTC, the risk of malignancy for an AUS nodule is difficult to ascertain because only a minority of cases in this category have surgical follow-up [3]. Among surgically managed cases $(n=100)$, more than half, $54 \%(n=54)$, were found to have thyroid cancer on histopathological analysis. This finding is similar to a recent study done in the same region [5]. This could be explained by the common genetic pool in Middle Eastern population. More importantly, however, the high incidence of malignancy can be explained by the referral bias in our study as it was conducted in a tertiary center where cases with suspicious thyroid nodules would be referred from most centers in the region. Previous literature demonstrates a variable rate of malignancy in this category, ranging from a rate as low as $6 \%$, as estimated by the BSRTC, to a rate as high as $69 \%$ in other studies [3, 14, 15]. Local data of 2 studies demonstrated around $18 \%$ prevalence of malignancy in Bethesda III ( 6 out of 32 cases) [16] and 20\% when UK classification is used and intermediate thyroid nodules were analyzed [17].

One study suggested the reason behind the great variability in the results stems from several factors as follows: 
(1) interobserver variability in the interpretation; (2) variations in specimen preparation methods; and (3) selection bias as not all AUS nodules were followed by histopathological diagnosis to estimate the overall incidence of malignancy [18]. Due to all these reasons, caution should be exercised when establishing an institution-specific or population-specific malignancy risk in Bethesda III category.

Among biopsied cases, papillary thyroid cancer was the most common type of malignancy. It was seen in 39 patients (72.2\%). This finding is comparable to other studies showing similar incidence rate of papillary cancer $(66.7 \%$, $n=84$ and $64.9 \%, n=24)$ and others showing a much higher rate $(90 \%, n=126)[9,15,19]$. Follicular cancer was the second most common, being found in 13 cases (24.1\%). This distribution in the incidence of papillary and follicular cancer is proportionate to other studies $[9,10,20]$.

The median size of the tumors was $1.25 \mathrm{~cm}$, and the range was between 0.1 and $8 \mathrm{~cm}$. The average size in our study is smaller than other reported studies with average size of $2.34 \pm 0.94 \mathrm{~cm}(0.8-6 \mathrm{~cm})$ [10]. None of the factors, including age, sex, or nationality, determined the outcomes of Bethesda III lesions after surgery. Other studies found a significant correlation between risk of malignancy and age where patients with malignant outcome were younger $(p<0.01)$ and had smaller thyroid nodules $(p<$ 0.05 in one study and $p<0.01$ in another study) $[10,21]$. The finding of smaller nodules positive correlation with risk of malignancy contradicted other previous studies that concluded that increased size impacted cancer risk in a nonlinear fashion [22].

Some studies have attempted to risk stratify Bethesda III according to specific cytopathological findings like exhibiting nuclear atypia versus those with architectural atypia [20]. The study demonstrated that nuclear atypia was associated with a higher rate of malignancy than architectural atypia $(p<0.05)$. TBSRTC updated guidelines supports the findings in this study by recommending laboratories to subcategorize the findings in AUS cytology according to nuclear atypia, architectural atypia, oncocytic features, and not otherwise specified. These four categories are suggested to guide the management of AUS nodules with nuclear atypia having the highest risk of malignancy $[3,23]$. In our current study, however, further cytological details were not provided.

Other studies have correlated the FNA results and histopathological diagnosis with ultrasound features (K-TIRADs) and concluded that malignant nodules were associated with higher ultrasound score, specifically with the presence of hypo- or anechogenicity in AUS/FLUS nodules [21]. Our study is limited by several factors as fol- lows: first, the retrospective nature of the study which resulted in recall bias and missing of some data. Second, the study was conducted in a tertiary center which potentially has resulted in a referral bias where only suspicious thyroid nodules would be referred to our service.

\section{Conclusion}

The finding highlighted in our study, which is supported by several previous studies, suggests a higher incidence of malignancy in Bethesda III category than the TBSRTC estimate. It also puts in question the utility and benefit of keeping a time gap and repeating FNA as previously recommended.

We propose further larger studies assessing more time efficient methods of categorizing and risk stratifying patients under this category, such as immunohistochemistry and molecular analysis methods. We hope that with an accurate risk stratification method, we would avoid time delays and missing cases with malignancy as well as avoid unnecessary surgical intervention in cases with benign pathology.

\section{Statement of Ethics}

Study protocol was approved by Dubai Local Ethical Committee. Reference number: DSREC-06/2016_04. According to that, data security and patient's confidentiality were maintained throughout the study.

\section{Conflict of Interest Statement}

All authors declare no conflicts of interest.

\section{Funding sources}

Study did not receive any funding.

\section{Author Contributions}

M.O.S.: study idea development, data collection, revision and analysis, and writing of manuscript. A.A.K.: study concept and development, data collection, writing of manuscript, and critical revision of manuscript. F.A.: study concept, data collection, critical revision of manuscript, and overall supervision of study process. M.A.: study concept, data collection, critical revision of manuscript, and supervision. A.B.: study concept and development, data collection, writing of manuscript, and critical revision of manuscript. 


\section{References}

1 Guth S, Theune U, Aberle J, Galach A, Bamberger CM. Very high prevalence of thyroid nodules detected by high frequency $(13 \mathrm{MHz})$ ultrasound examination. Eur J Clin Invest. 2009 Aug 1;39(8):699-706.

2 Cibas ES, Ali SZ. NCI thyroid FNA state of the science conference. The Bethesda System for reporting thyroid cytopathology. Am J Clin Pathol. 2009 Nov 1;132(5):658-65.

3 Cibas ES, Ali SZ. The 2017 Bethesda System for reporting thyroid cytopathology. Thyroid. 2017 Nov 1;27(11):1341-6.

4 Bongiovanni M, Spitale A, Faquin WC, Mazzucchelli L, Baloch ZW. The Bethesda System for reporting thyroid cytopathology: a metaanalysis. Acta Cytol. 2012;56(4):333-9.

5 Zarif HA, Ghandurah SE, Al-Garni MA, Binmahfooz SK, Alsaywid BS, Satti MB. Thyroid nodules cytopathology applying the Bethesda System with histopathological correlation. Saudi J Med Med Sci. 2018 Dec 1;6(3):143-8.

6 Almahari SA, Harb Z, Alshaikh S. Evaluation of thyroid nodules classified as Bethesda category III on cytology and their malignancy rate: an institutional experience. Cytojournal. 2019 Sep 16;16:18.

7 Haugen BR. 2015 American thyroid association management guidelines for adult patients with thyroid nodules and differentiated thyroid cancer: what is new and what has changed?. Cancer. 2017 Feb 1;123(3):372-81.

8 Jiang $\mathrm{H}$, Tian $\mathrm{Y}$, Yan W, Kong Y, Wang H, Wang A, et al. The prevalence of thyroid nodules and an analysis of related lifestyle factors in Beijing communities. Int J Environ Res Public Health. 2016 Apr 22;13(4):442.

9 Maity P, Jha AK, Sengupta M, Basu K, Chatterjee U, Ghosh S. Thyroid Bethesda atypia of undetermined significance or follicular lesion of undetermined significance (AUS/FLUS): a heterogenous group. J Cytol. 2019 Oct-Dec 1; 36(4):200-4.
10 Mileva M, Stoilovska B, Jovanovska A, Ugrinska A, Petrushevska G, Kostadinova-Kunovs$\mathrm{ka} S$, et al. Thyroid cancer detection rate and associated risk factors in patients with thyroid nodules classified as Bethesda category III. Radiol Oncol. 2018 Sep 27;52(4):370-6.

11 Guo A, Kaminoh Y, Forward T, Schwartz FL, Jenkinson S. Fine needle aspiration of thyroid nodules using the Bethesda System for reporting thyroid cytopathology: an institutional experience in a rural setting. Int J Endocrinol. 2017;2017:9601735.

12 Seagrove-Guffey MA, Hatic H, Peng H, Bates $\mathrm{KC}$, Odugbesan AO. Malignancy rate of atypia of undetermined significance/follicular lesion of undetermined significance in thyroid nodules undergoing FNA in a suburban endocrinology practice: a retrospective cohort analysis. Cancer Cytopathol. 2018 Oct 1; 126(10):881-8.

13 Thewjitcharoen Y, Butadej S, Nakasatien S, Chotwanvirat P, Porramatikul S, Krittiyawong $S$, et al. Incidence and malignancy rates classified by The Bethesda System for Reporting Thyroid Cytopathology (TBSRTC): an 8-year tertiary center experience in Thailand. J Clin Transl Endocrinol. 2018 Dece 14;16: 100175.

14 Chandra S, Chandra H, Bisht SS. Malignancy rate in thyroid nodules categorized as atypia of undetermined significance or follicular lesion of undetermined significance: an institutional experience. J Cytol. 2017 Sep 1;34(3): 144-8.

15 Park JH, Yoon SO, Son EJ, Kim HM, Nahm $\mathrm{JH}$, Hong S. Incidence and malignancy rates of diagnoses in the bethesda system for reporting thyroid aspiration cytology: an institutional experience. Korean J Pathol. 2014 Apr 1;48(2):133-9.

16 Alseddeeqi E, Baharoon R, Mohamed R, Ghaith J, Al-Helali A, Ahmed LA. Thyroid malignancy among patients with thyroid nodules in the United Arab Emirates: a fiveyear retrospective tertiary Centre analysis. Thyroid Res. 2018 Nov 30;11:17.
17 Khalil AB, Dina R, Meeran K, Bakir AM, Naqvi S, Al Tikritti A, et al. Indeterminate thyroid nodules: a pragmatic approach. Eur Thyroid J. 2018;7(1):39-43.

18 VanderLaan PA, Marqusee E, Krane JF. Clinical outcome for atypia of undetermined significance in thyroid fine-needle aspirations: should repeated fna be the preferred initial approach?. Am J Clin Pathol. 2011 May 1; 135(5):770-5.

19 Godoi Cavalheiro B, Kober Nogueira Leite A, Luongo de Matos L, Palermo Miazaki A, Marcel Ientile J, Aurelio V Kulcsar M, et al. Malignancy rates in thyroid nodules classified as Bethesda categories III and IV: retrospective data from a tertiary center. Int J Endocrinol Metab. 2018 Dec 13;16(1):e12871.

20 Gan TR, Nga ME, Lum JH, Wong WM, Tan WB, Parameswaran R, et al. Thyroid cytology-nuclear versus architectural atypia within the "Atypia of undetermined significance/follicular lesion of undetermined significance" Bethesda category have significantly different rates of malignancy. Cancer Cytopathol. 2017 Apr 1;125(4):245-56.

21 Hong SH, Lee H, Cho MS, Lee JE, Sung YA, Hong YS. Malignancy risk and related factors of atypia of undetermined significance/follicular lesion of undetermined significance in thyroid fine needle aspiration. Int J Endocrinol. 2018 Jul 30;2018:4521984.

22 Kamran SC, Marqusee E, Kim MI, Frates MC, Ritner J, Peters H, et al. Thyroid nodule size and prediction of cancer. J Clin Endocrinol Metab. 2013 Feb 1;98(2):564-70.

23 Iskandar ME, Bonomo G, Avadhani V, Persky M, Lucido D, Wang B, et al. Evidence for overestimation of the prevalence of malignancy in indeterminate thyroid nodules classified as Bethesda category III. Surgery. 2015 Mar 1;157(3):510-7. 